\title{
Gelation as Studied by Proton Multiple-Quantum NMR
}

\author{
Kay Saalwächter* \\ Institut für Physik, Martin-Luther-Universität Halle-Wittenberg, \\ Friedemann-Bach-Platz 6, D-06018 Halle, Germany

\section{Moshe Gottlieb} \\ The Raimund Stadler Minerva Center for Mesoscale Macromolecular Engineering, \\ and Department of Chemical Engineering, Ben-Gurion University of the Negev, \\ Beer-Sheeva 84105, Israel
}

\author{
Ruigang Liu ${ }^{\dagger}$ and Wilhelm Oppermann \\ Institut für Physikalische Chemie, Technische Universität Clausthal, \\ Arnold-Sommerfeld-Str. 4, D-38678 Clausthal-Zellerfeld
}

Received December 4, 2006; Revised Manuscript Received January 11, 2007

\begin{abstract}
A novel NMR approach for the study of gelation in flexible polymer systems is presented, and the data are compared to results from rheological and light scattering experiments. Proton multiple-quantum NMR, also applicable at low field and amenable to high-throughput experimentation, represents a simple and robust yet powerful method that allows one to detect the topological gel point, quantify the gelation kinetics, and characterize composition, microstructure, and dynamics of the forming and the final gel. Applications to end-linked and statistically cross-linked poly(dimethylsiloxane) chains in the bulk, as well as statistically cross-linked poly(styrene) chains in solution are presented, and good agreement as well as complementarity between the results from the different methods is found.
\end{abstract}

\section{Introduction}

Gelation phenomena are abundant in polymeric systems, and their understanding is a matter of vast significance for industrial applications as well as academia. ${ }^{1}$ The topological gel point, defined as the extent of reaction above which a percolated, infinite network forms, may be estimated by a number of simple mechanical tests. On the other hand, the understanding of how the gel point is reflected quantitatively in the rheological response or more local structural and dynamic properties, as for instance measured in scattering experiments, has been a matter of active research. ${ }^{1-8}$

Using low-amplitude oscillatory shear experiments, Chambon and Winter first observed that the storage and loss moduli of bulk gelling systems follow the same power-law behavior at the gel point over large frequency ranges, over which the loss tangent $\tan \delta=G^{\prime \prime} / G^{\prime}$ is consequently independent of frequency. ${ }^{2-4}$ The power-law exponent, which supposedly depends on the fractal dimension of the incipient gel, was later related to the power-law exponent found in the time correlation function of dynamic light scattering experiments. ${ }^{5,6}$ In the latter type of experiments, the gel point can be defined as the extent of reaction above which such a power law is detected. It is accompanied by the appearance of strong spatial intensity fluctuations (speckles), indicating the loss of ergodicity. ${ }^{7,8}$ In this paper, an NMR approach is presented that gives results for the gel point that are consistent with rheology and light scattering while being technically undemanding and quick.

The gel point is not straightforwardly reflected in NMR in general. While it is of course easy to monitor the extent of

* Corresponding author. E-mail: kay.saalwaechter@physik.uni-halle. de. URL: www.physik.uni-halle.de/nmr.

$\dagger$ Now at Key Laboratory of Polymer Physics and Chemistry, Institute of Chemistry, The Chinese Academy of Sciences, Zhongguancun North First Street 2, 100080 Beijing, China. reaction via observing the chemical conversion in a network forming system by means of methods providing chemical-shift resolution, ${ }^{9,10}$ an observable that exhibits the required sensitivity to the specific changes in the dynamics of a percolating structure is harder to identify. High-resolution $T_{1}$ relaxation $^{11}$ and diffusion $\mathrm{NMR}^{12}$ have been applied to detect changes in the very fast short-range and very slow long-range mobility, respectively, of the gelling component and were found to exhibit a correlation with the gel point. A general limitation of highresolution proton NMR is, however, that the actually formed gel is not quantitatively detected as it features very broad lines due to rather short transverse relaxation times.

The proton $T_{2}$ behavior has long been known to reflect structure and dynamics of mobile polymer networks and gels ${ }^{13-15}$ and should exhibit a better sensitivity to the actual ( $\mu$ s to $\mathrm{ms}$ ) time scale of chain fluctuations related to entanglements or cross-links, and thus to the gel point. In fact, in very early work, the appearance of an additional, shorter $T_{2}$ component associated with the network was correlated with the gel point, ${ }^{13}$ and more recent examples of the observation of qualitative changes in the $T_{2}$ behavior upon gelation can be found in refs 16 and 17 . We here present a method that is based on similar principles, but features a direct single-point detection of the gel point and thus avoids the measurement and decomposition of complete relaxation curves.

We make use of the fact that the NMR response of network systems is partially solid-like. In isotropically mobile polymer solutions or melts, orientation-dependent interactions that would dominate the spectral response of rigid solids are averaged out completely and are thus undetectable. Yet, chain segment fluctuations are not completely isotropic in networks because of the constraints posed by the cross-links, leaving weak residual orientation-dependent interactions that lead to coherent dipolar dephasing. ${ }^{18}$ This phenomenology is often confused with transverse relaxation. 
An important feature is that residual interactions are generally fluctuating quantities that give rise to a complex interplay of dephasing and relaxation effects, but become stable over long observation times upon transition from linear polymers (or monomers) to an infinite gel structure, where they are known to reflect the macroscopic elastic properties of permanent networks. ${ }^{19}$ We thus focus on the direct detection of residual proton-proton dipolar couplings and show that they constitute a sensitive means to monitor the gelation process in a quantitative fashion. This includes the determination of the gel point, gelation kinetics, and the microstructural characterization of the gelling system. As to be demonstrated, NMR is in this regard complementary to rheological or scattering methods, yet the method proposed here relies on simple low-field instrumentation with only minimal demands on sample preparation, and it simultaneously provides access to a number of important variables such as the chain length between cross-links and the composition of the gelling sample.

A variety of NMR experiments, most of them based on spin echoes, has been used over the last decades to detect residual proton dipolar interactions in polymeric systems ${ }^{14,20-25}$ and also to monitor the gelation process. ${ }^{26-28}$ In this respect, CohenAddad's "pseudosolid", spin echoes, which are a direct means to detect residual couplings, have been shown to qualitatively reflect the cross-linking process. ${ }^{26}$ Recently, proton multiplequantum (MQ) $\mathrm{NMR}^{29}$ has evolved as an even more powerful tool. ${ }^{30-32}$ While the most elementary realizations of this type of spectroscopy are even applicable in the highly inhomogeneous field of single-sided NMR surface probes, ${ }^{33}$ we could show that an alternative approach provides quantitative information on structure and even heterogeneities (i.e., distributions) ${ }^{32,34-36}$ as well as chain dynamics. ${ }^{37,38}$ Some artifacts that may arise when simpler NMR methods are used are thus avoided. ${ }^{39,40}$

The experiment is based on a robust and easily implementable (yet lengthy) pulse sequence 29,32 that can be performed on lowfield NMR analyzers in an automated fashion without any sacrifice in data quality. ${ }^{36,41}$ For the first time, we here apply MQ NMR to the study of the gelation process, compare the results with established methods such as rheology and dynamic light scattering (DLS), and discuss its advantages and limitations.

\section{Experimental}

Samples. Vinyl-terminated PDMS (PDMS424, $M_{\mathrm{n}} \approx$ $5000 \mathrm{~g} / \mathrm{mol}$ ) and randomly vinyl-functionalized PDMS (PDMS441, $M_{\mathrm{n}} \approx 15000 \mathrm{~g} / \mathrm{mol}$ ) were cross-linked using tetrakis(dimethylsiloxy)silane as a cross-linker and cis-dichlorobis(diethylsulfato)platinum(II) in $2 \%$ toluene solution as the catalyst. All materials were purchased from United Chemical Technologies Inc. (Bristol, PA) and used as received. On the basis of the average functionalities as derived from solution-state ${ }^{1} \mathrm{H}$ NMR spectra, different mixtures using a given stoichiometric ratio $r$ of silane to vinyl groups were prepared and cured directly in the rheometer and later used for NMR spectroscopy.

Polystyrene $\left(M_{\mathrm{w}}=3.35 \times 10^{5} \mathrm{~g} / \mathrm{mol}\right.$ and $\left.M_{\mathrm{w}} / M_{\mathrm{n}}=2.1, \mathrm{BASF}\right)$ was randomly functionalized to obtain poly(styrene-co-aminomethylstyrene) [P(S-co-AMS $)]$ following a well-described synthetic procedure. ${ }^{42}$ The mole ratio of aminomethyl groups and styrene repeat units was 1:27. The amine groups of the copolymer can be linked via dialdehydes to form a network. For the light scattering experiments, solutions containing $0.1-0.5 \mathrm{~g}$ of $\mathrm{P}(\mathrm{S}-\mathrm{co}-\mathrm{AMS})$ in 8 $\mathrm{mL}$ of toluene (spectroscopy grade, Aldrich) were prepared. Likewise, solutions of 2.15-10.74 mg of terephthaldialdehyde in $2 \mathrm{~mL}$ of toluene were made. Corresponding solutions were filtered through a $1 \mu \mathrm{m}$ PTFE membrane directly into a dust-free light scattering cuvette and mixed to obtain a total volume of $10 \mathrm{~mL}$. DLS measurements were started immediately thereafter. For the
FID detection

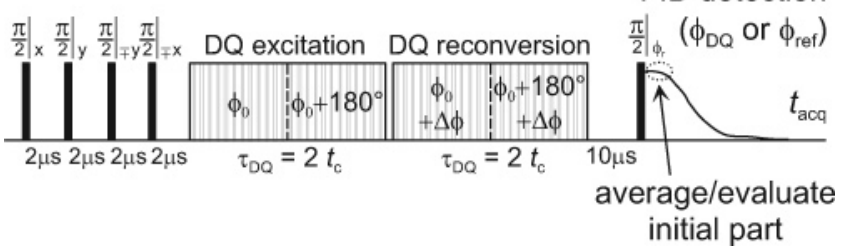

Figure 1. Pulse sequence for MQ spectroscopy on a Bruker minispec. The DQ excitation and reconversion blocks are identical and consist of two cycles each of the pulse sequence described in ref 32. Their base phase $\phi_{0}$, to which the specific pulse phases are to be added, is inverted for the second cycle. The DQ selection phase cycle is realized via the increment $\Delta \phi$. The initial four $90^{\circ}$ pulses comprise a robust and accurate composite-pulse inversion that is controlled by the phase cycle.

NMR experiments, stock solutions of $625 \mathrm{mg} \mathrm{P}(\mathrm{S}-\mathrm{co}-\mathrm{AMS})$ and $26.75 \mathrm{mg}$ terephthaldialdehyde in $10 \mathrm{~mL}$ and $5 \mathrm{~mL}$ of toluene- $d_{8}$, respectively, were prepared. These were combined in the stoichiometric volume ratio of $4: 1$ in NMR tubes $(\Longrightarrow c=0.05 \mathrm{~g} / \mathrm{mL})$, diluted as required to always give a final volume of $1 \mathrm{~mL}$ in a $10 \mathrm{~mm}$ OD NMR tube, shaken quickly, and transferred to the spectrometer within less than $1 \mathrm{~min}$.

Rheology. Oscillatory shear measurements were carried out at room temperature with a CarriMed CLS50 constant stress rheometer (CarriMed Instruments Ltd., Dorking, U.K.) using a cone-and-plate geometry.

Dynamic Light Scattering. DLS measurements were performed at $25{ }^{\circ} \mathrm{C}$ on an ALV/CGS-3 compact goniometer (ALV, Langen, Germany) equipped with a $\mathrm{He}-\mathrm{Ne}$ laser $\left(22 \mathrm{~mW}, \lambda_{0}=632.8 \mathrm{~nm}\right)$. The scattering intensity was collected at a scattering angle of $90^{\circ}$ as a function of reaction time. The integration time interval of the detection system was set to $20 \mathrm{~s}$.

NMR Spectroscopy. ${ }^{1} \mathrm{H}$ MQ experiments were performed on a Bruker minispec mq20 $\left(B_{0}=0.5 \mathrm{~T}\right)$ and evaluated following previously published procedures. ${ }^{32,35}$ The sample temperature was maintained using a regulated airflow and kept at $25 \pm 1{ }^{\circ} \mathrm{C}$ unless noted otherwise. The tune-up is simple and requires only the proper adjustment of the $90^{\circ}$ pulse length (typically about $1.8-2 \mu \mathrm{s}$ ) and the offset as well as the receiver phase to obtain a monotonically decaying on-resonance real FID with negligible contributions in the imaginary (sine) part. The samples were kept in $10 \mathrm{~mm}$ OD NMR tubes, and their height was $7 \mathrm{~mm}$ at most, with the center located in the center of the $\mathrm{rf}$ coil to ensure sufficient $B_{1}$ homogeneity.

For an in-depth discussion of data acquisition and analysis, we refer the reader to previous papers. ${ }^{32,35,36} \mathrm{We}$ here mainly give some details that are relevant for the actual implementation on the minispec (see Figure 1). Generally, the experiment yields a DQ intensity build-up, $I_{\mathrm{DQ}}\left(\tau_{\mathrm{DQ}}\right)$, that is dependent on the evolution time under a pulse sequence with a pure DQ average Hamiltonian and reflects the magnitude of homonuclear residual dipolar couplings. The overall DQ evolution time, $\tau_{\mathrm{DQ}}$, is incremented by increasing the interpulse spacings that are given in ref 32 .

One should keep in mind that even though the experimental procedure comprises the excitation of multiple higher quantum orders, all arguments are restricted to double-quantum (DQ) coherences, as only these reach significant intensities. They were selected by a four-step phase cycle on $\Delta \phi=\{x, y,-x,-y\}$, accompanied by receiver phase inversion for every other transient: $\phi_{\mathrm{DQ}}=\{x,-x, x,-x\}$. The reference intensity, $I_{\mathrm{ref}}\left(\tau_{\mathrm{DQ}}\right)$, which contains information on the sol content and can further be used to correct for relaxation effects under the pulse sequence, is obtained in an independent experiment by using $\phi_{\mathrm{ref}}=\{x, x, x, x\}$ as the receiver phase. On top of this, four-step CYCLOPS was performed on the read pulse $\left(\phi_{\mathrm{r}}\right)$ and the receiver phases.

At $20 \mathrm{MHz}$ Larmor frequency, $T_{1}$ relaxation times of mobile polymer systems often reach the ms range. To suppress intensity contributions arising from the reappearance of the signal during the course of the experiment, a two-step phase-cycle controlled 
polarization inversion was applied in addition, yielding a total of 32 transients per experiment. The final intensities were automatically evaluated from an average over the initial $100 \mu$ s of the FID, during which the signal does not decay appreciably. A gain in signal-tonoise would of course be possible by using the whole FID or even lengthening it artificially by a pulsed spin lock, but this was not necessary for the data presented herein. Usually, between 32 (bulk samples) and 512 transients (dilute solutions) were recorded for the measurement of a single point, using recycle delays ranging between $200 \mathrm{~ms}$ to $1 \mathrm{~s}$ (longer waiting times were needed when sol or solvent exhibit a longer $T_{1}$ in order to obtain the correct signal ratio).

The sum of the DQ and reference intensities, possibly corrected by mobile components $\mathrm{B}$,

$$
I_{\Sigma \mathrm{MQ}}=I_{\mathrm{DQ}}+I_{\mathrm{ref}}-B e^{-2 \tau_{\mathrm{DQ}} / T_{2 \mathrm{~B}} *}
$$

is analogous to a full (dipolar and chemical-shift) echo that is only subject to dipolar-induced relaxation decay. ${ }^{37,38}$ All data in this paper is plotted on a scale defined by $I_{\mathrm{DQ}}+I_{\mathrm{ref}}=1$ for $\tau_{\mathrm{DQ}}=$ 0 , i.e., the full magnetization of the sample detected after a $90^{\circ}$ pulse. $I_{\Sigma \mathrm{MQ}}\left(\tau_{\mathrm{DQ}}\right)$ can be used to normalize the experimental DQ build-up point by point in order to remove relaxation effects:

$$
I_{\mathrm{nDQ}}\left(\tau_{\mathrm{DQ}}\right)=I_{\mathrm{DQ}}\left(\tau_{\mathrm{DQ}}\right) / I_{\Sigma \mathrm{MQ}}\left(\tau_{\mathrm{DQ}}\right)
$$

In networks and gels at temperatures exceeding $T_{\mathrm{g}}$ by at least 50 $\mathrm{K}$, this procedure can be refined by subtraction of one or two exponential long-time contributions to $I_{\Sigma \mathrm{MQ}}$ (fraction $B$ in eq 1) that reflect solvent, sol, and/or dangling chains. See refs 32, 35 for a discussion of the limitations of the normalization approach and a detailed description of the fitting strategy. When mobile components are properly taken into account, $I_{\mathrm{nDQ}}$ is temperature-independent and only depends on residual dipolar couplings ( $\left.D_{\text {res }}\right)$. The couplings arise as a consequence of the topological restrictions of the network chains, are proportional to the local cross-link density, and consequently inversely related to the length of a chain between restrictions. Importantly, the distribution is also accessible and carries information about heterogeneities. ${ }^{32,36}$ Most networks we have as yet investigated exhibit surprisingly narrow distributions of $D_{\text {res}}$, such that the second-moment approximation formula of a single-coupling scenario can be used for fitting:

$$
I_{\mathrm{nDQ}}\left(D_{\mathrm{res}}\right)=\frac{1}{2}\left(1-\exp \left\{-\frac{2}{5} D_{\mathrm{res}}{ }^{2} \tau_{\mathrm{DQ}}{ }^{2}\right\}\right)
$$

As this is an approximation, only experimental data for $I_{\mathrm{nDQ}} \leq 0.45$ are considered for fitting. ${ }^{38}$ Note that $D_{\text {res }}$ is of course an apparent coupling constant and represents an average over many different internuclear pair couplings. Marked deviations of the build-up data from an inverted Gaussian indicate the presence of a substantial distribution. Assuming this distribution to be Gaussian, ${ }^{32}$ the relation

$$
I_{\mathrm{nDQ}}\left(D_{\mathrm{G}}, \sigma_{\mathrm{G}}\right)=\frac{1}{2}\left(1-\frac{\exp \left\{-\frac{\frac{2}{5} D_{\mathrm{G}}{ }^{2} \tau_{\mathrm{DQ}}{ }^{2}}{1+\frac{4}{5} \sigma_{\mathrm{G}}{ }^{2} \tau_{\mathrm{DQ}}{ }^{2}}\right\}}{\sqrt{1+\frac{4}{5} \sigma_{\mathrm{G}}{ }^{2} \tau_{\mathrm{DQ}}{ }^{2}}}\right)
$$

can be used to obtain results for the average coupling constant as well as the standard deviation $\left(D_{\mathrm{G}}\right.$ and $\sigma_{\mathrm{G}}$, respectively). It is important to realize that fits to this function make physical sense only as long as $\sigma_{\mathrm{G}}$ is substantially smaller than $D_{\mathrm{G}}$. Numerical inversion procedures can otherwise be used to assess the distribution. ${ }^{32}$ For an in-depth discussion of the relationship between $D_{\text {res }}$ and the actual cross-link density, see ref 36.

As will become evident below, a fully quantitative gel characterization is restricted to systems well above the gel point. During the course of gelation, the system is composed of polydisperse com-

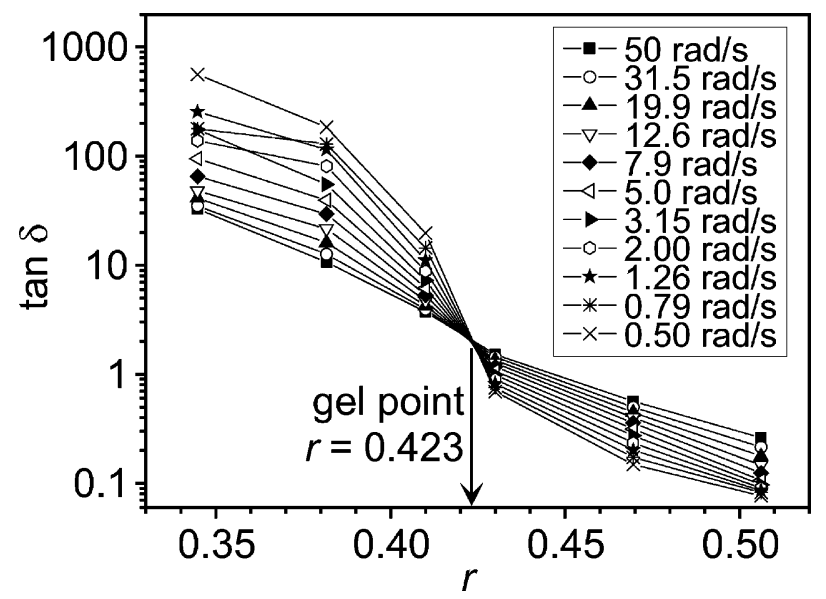

Figure 2. Rheological determination of the gel point of end-linked PDMS441 following the method of Winter and Chambon. ${ }^{2,3}$

ponents with different mobility such that the mobile-component subtraction, as well as the normalization, can be subject to ambiguities.

\section{Results and Discussion}

In the following, we present NMR investigations of three conceptually different gelling systems, i.e., bulk end-linking of linear PDMS chains (PDMS441), bulk random cross-linking (vulcanization) of PDMS containing random vinyl functionalities (PDMS424), and random cross-linking of $\mathrm{P}(\mathrm{S}-\mathrm{co}$-AMS) in semidilute solution using deuterated toluene as solvent. For the first two cases, fully reacted systems were studied as a function of cross-linker concentration and are compared with rheological experiments, while for the latter, stoichiometric compositions were studied as a function of reaction time and are compared with DLS results. Note that PDMS424 and P(S-co-AMS) are similar in that they both are cross-linked at fixed but random positions along the chains.

A. Gelation of Bulk PDMS and Comparison with Rheology. Results of frequency-dependent determinations of the rheological loss tangent of samples from the end-linking series of PDMS441 are shown in Figure 2. The acquisition of an $\omega$ sweep for a single sample takes many hours compared to about $1 \mathrm{~min}$ (10 min including routine setup) on about $200 \mathrm{mg}$ or less of sample for the single-point NMR measurement discussed below. The mechanical gel point is readily identified as the silane to vinyl ratio $r$ for which $\tan \delta$ is independent of frequency.

Data from complete MQ NMR build-up/decay analyses of the same series are plotted in Figure 3. A full $\tau_{\mathrm{DQ}}$-dependent time series of a bulk sample can be acquired in about $1 \mathrm{~h}$ (phasecycle limited). Only above the mechanical gel point at $r=0.424$ is significant DQ intensity observed. This is in line with the notion that only chains that participate in a percolated crosslinked structure are fluctuating nonisotropically at temperatures that are sufficiently far above $T_{\mathrm{g}}$. The appearance of DQ intensity, i.e., the presence of a detectable fraction of such chains, is here defined as the NMR gel point.

For the most sensitive single-point measurement to be discussed below, we focus in the following on the normalized DQ intensity at a $\tau_{\mathrm{DQ}}$ for which $I_{\mathrm{nDQ}}$ or $I_{\mathrm{DQ}}$ attains its highest values (or reaches its plateau) in the fully cured gel, which occurs at about the same $\tau_{\mathrm{DQ}}$ when the temperature is sufficiently high and relaxation effects do not lead to more that $50 \%$ loss of gel fraction signal. This implies that a complete time series should be recorded at least for a fully cured/stoichiometric sample. In PDMS441, the maximum is observed at $\tau_{\mathrm{DQ}} \approx 5 \mathrm{~ms}$. 

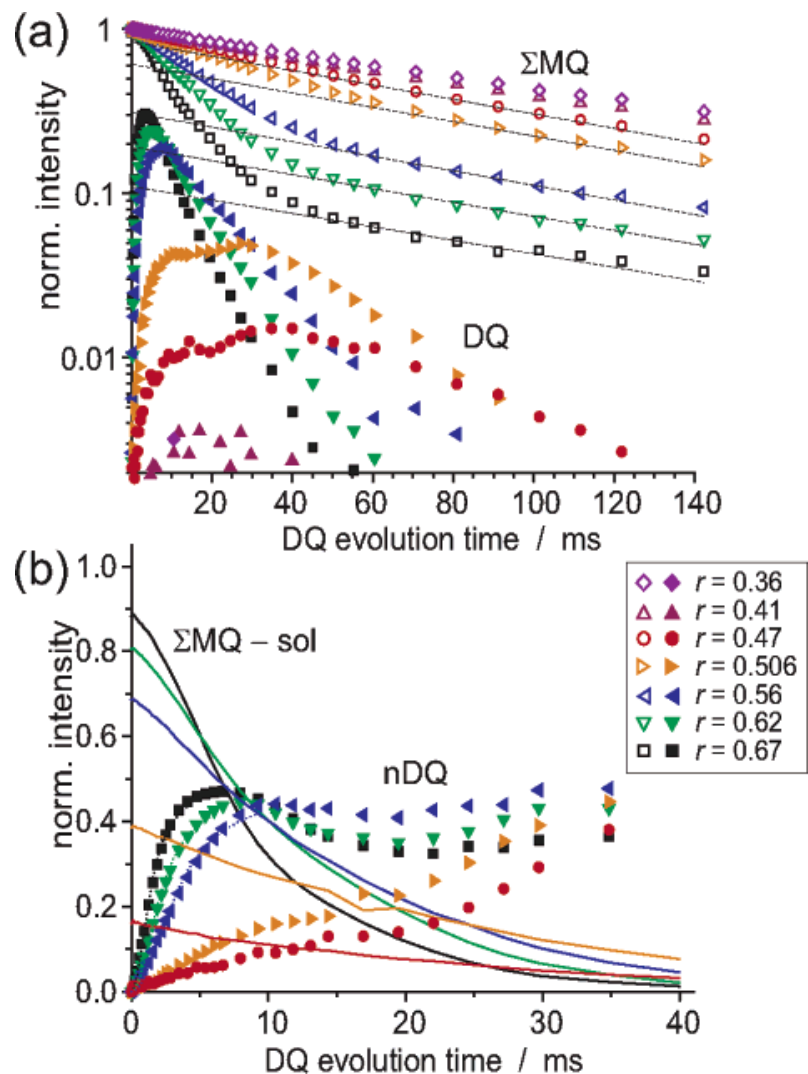

Figure 3. MQ NMR build-up and decay data for the gelation of endlinked PDMS441. (a) As-acquired $I_{\Sigma \mathrm{MQ}}$ and $I_{\mathrm{DQ}}$. The lines are exponential fits to $I_{\Sigma \mathrm{MQ}}$ for $\tau_{\mathrm{DQ}} \geq 50 \mathrm{~ms}$ that represent the sol contribution. (b) $I_{\Sigma \mathrm{MO}}$ (lines) and $I_{\mathrm{nDQ}}$ (symbols) after subtraction of the sol component. The fits of the nDQ curves for $r \geq 0.56$ to eq 4 are plotted as dotted lines.

The complete analysis offers rich insight into the gelation process. The full intensity data in Figure 3a show exponential tails of $I_{\Sigma \mathrm{MQ}}$ that represent the sol fraction. Moreover, for stoichiometries just above the gel point, $I_{\mathrm{DQ}}$ exhibits two maxima reflecting a heterogeneous gel structure. These maxima transform into a two-step build-up after point-by-point normalization (Figure $3 b$ ), as is expected when relaxation effects are successfully accounted for.

We infer that the more weakly coupled fraction, associated with more isotropically mobile (possibly longer) network chains, decreases at the expense of chains that ultimately form the percolated network. A more complete study would have to comprise temperature-dependent measurements in order to check whether the weakly coupled chains may be unconnected chemically extended chains or branched structures that acquire residual couplings as a result of physical entanglements. These may lose their stability on the experimental time scale when the temperature is raised. ${ }^{38}$

From Figure 3b, it is also clear that up to $50 \%$ of the whole sample magnetization of a system well above the gel point (excluding the sol) can be converted into DQ coherences using a $\tau_{\mathrm{DQ}}$ within which the overall magnetization has not decayed by more than about $30 \%$ (or even less when the temperature is raised). This shows that the DQ method is by no means insensitive. In fact, it is more difficult to perform a reliable $T_{2}$ decay component analysis than simply observing a DQ intensity that unambiguously reflects up to $50 \%$ of the overall intensity of the true network component.

The residual couplings derived from fits to eq 4 for the three samples with the highest $r$ are 60,90, and $150 \mathrm{~Hz}$, with standard
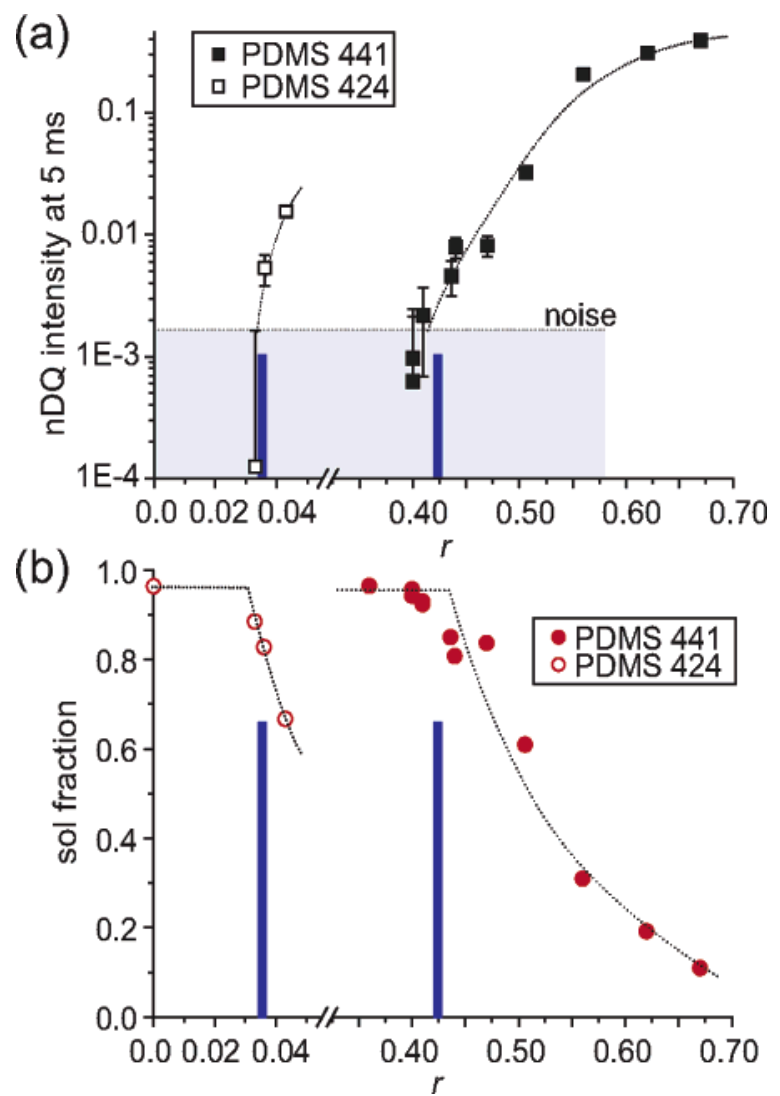

Figure 4. (a) Single-point detection of the gel point of end-linked PDMS441 and statistically cross-linked PDMS424, represented by $I_{\mathrm{nDQ}}(5 \mathrm{~ms})$. No sol correction was applied, and the shaded area indicates the noise level (equal to the experimental relative root-mean-square intensity deviation from zero $=0.0015$, and identical to the error bar size). (b) Sol fractions for the two sample series as obtained from the fits shown in Figure 3a. All dotted lines are guides to the eye, and the vertical bars indicate the rheological gel points.

deviations $\sigma_{\mathrm{G}}$ (distribution width) of about $50 \%$ of these values. In our previous work, ${ }^{32,39}$ we have found a value of $\sim 360 \mathrm{~Hz}$ for a fully cured end-linked PDMS with $M_{\mathrm{c}} \approx 5000 \mathrm{~g} / \mathrm{mol}$, yet with a different end-linking chemistry that might interfere with the chain length distribution. ${ }^{43} \mathrm{We}$ might nevertheless speculate that, at the given maximum stoichiometric ratio, chain extension is still significant.

We now turn to the results for single-point NMR gel point determination for both the end-linked PDMS441 as well as the statistically cross-linked PDMS424 that are summarized in Figure 4a. The mechanically detected gel points are denoted as vertical bars, and their location closely coincides with the rise of observable DQ intensity, thus showing that the gel point definitions of two methods are compatible for the systems at hand and are thus sensitive to similar topological features of the gels. From Figure 4b, we further infer that the sol content (of course not available from a single-point measurement) also shows a significant drop at and beyond the gel point. Note that the observation of somewhat less than $100 \%$ sol even below the gel point is due to the slightly nonexponential $\Sigma$ MQ decay function even for the pure precursor polymers (as indicated by the $r=0$ point for PDMS 424 in Figure 4b). Similar observations were reported in an NMR diffusion study of gelatin, where the fraction of diffusing sol was also found to decrease just beyond the gel point. ${ }^{12}$

B. Gelation of Dilute PS Solutions and Comparison with DLS. Observing gelation in a rather dilute system (from the NMR point of view) is a challenge in particular for insensitive 

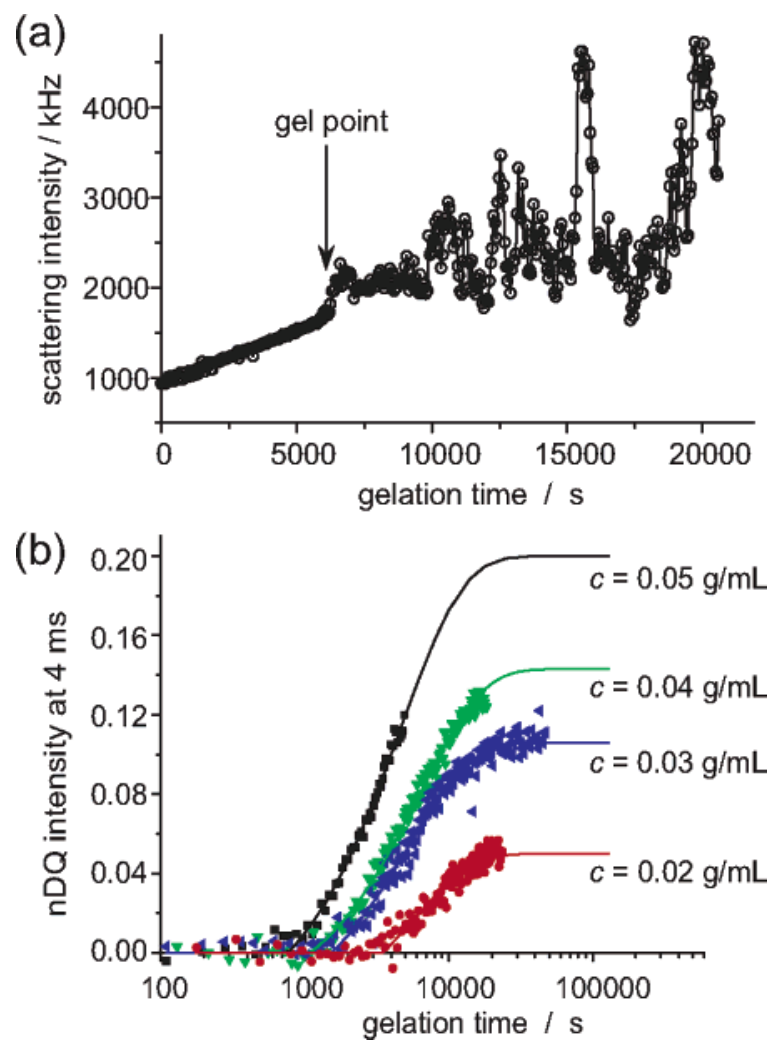

Figure 5. Gelation kinetics of dilute solutions of $\mathrm{P}(\mathrm{S}$-co-AMS) in toluene- $d_{8}$ cross-linked with terephtaldialdehyde. (a) Gel point as inferred from the appearance of a speckle pattern in DLS $(c=0.02$ $\mathrm{g} / \mathrm{mL}$ ). (b) NMR gelation kinetics derived from real-time single-point measurements of $I_{\mathrm{nDQ}}(4 \mathrm{~ms})$ (no sol correction) at different concentrations. The exponential fits (solid lines) yield onset (gel point) times and gelation time constants. The final $I_{\mathrm{nDQ}}(4 \mathrm{~ms})$ was measured $24 \mathrm{~h}$ after completion of the kinetics experiment and was held constant for the fits.

low-field NMR equipment. The ensuing results show this is nevertheless possible when the experiment is based on a singlepoint observable. We focus on the gelation kinetics of dilute PS chains with stoichiometric amounts of cross-linker, where the time resolution was on the order of 2 min (note that for each nDQ point, two intensities, DQ and ref, are measured).

Results from real-time DLS and NMR experiments are compared in Figure 5. The DLS gel point is linked to the appearance of strong intensity fluctuations, related to a speckle pattern that arises when the system becomes nonergodic on the time scale of the $20 \mathrm{~s}$ integration time interval of the detection system. ${ }^{7,8}$ In Figure 5b, this is contrasted with the time dependence of $I_{\mathrm{nDQ}}(4 \mathrm{~ms})$ measured for gelation at different concentrations. $\tau_{\mathrm{DQ}}=4 \mathrm{~ms}$ is again based on build-up experiments on fully cured gels, see below.

The so-obtained data could be well fitted to an exponential build-up as a function of the gelation time, $t$, and featuring a lag time, $t_{0, \mathrm{NMR}}$, that represents the gel point time:

$$
\begin{aligned}
I_{\mathrm{nDQ}}\left(\tau_{\mathrm{max}}\right)= & 0 \text { for } t<t_{0, \mathrm{NMR}} \\
I_{\mathrm{nDQ}}\left(\tau_{\mathrm{max}}\right)= & I_{\mathrm{nDQ}}\left(\tau_{\max }\right)_{t \rightarrow \infty} \times\left(1-e^{-t / \tau_{\mathrm{NMR}}}\right) \\
& \text { for } t \geq t_{0, \mathrm{NMR}}
\end{aligned}
$$

The second accessible parameter is the apparent gelation time constant, $\tau_{\mathrm{NMR}}$, the inverse of which represents the build-up rate of cross-link density.

Both quantities are plotted and compared with the DLS results in Figure 6. It is apparent that here, NMR detects gelation earlier

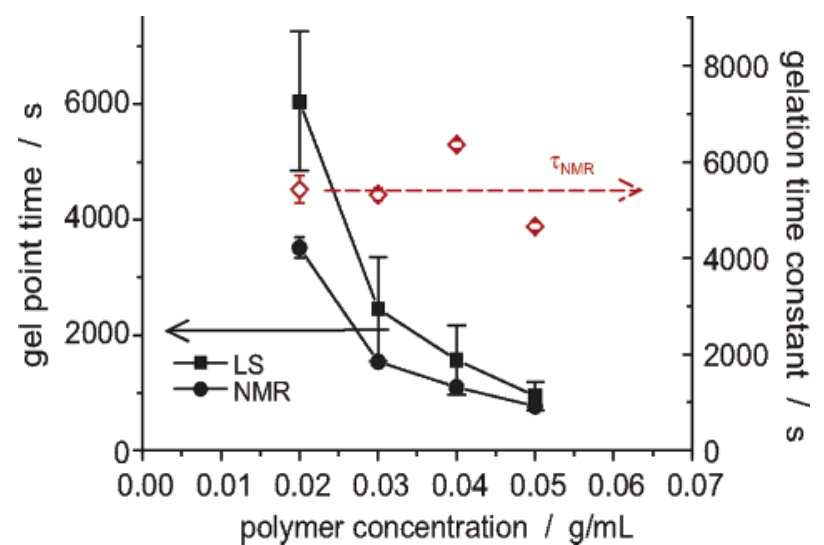

Figure 6. Comparison of the gel point times of $\mathrm{P}(\mathrm{S}-\mathrm{co}$-AMS) in toluene from DLS and NMR experiments, as well as the NMR-determined gelation time constants. The DLS data differ somewhat from those given in ref 8 , as the present study was carried out on different new batches of polymer.

than DLS. It must be noted that there is some degree of uncertainty in this comparison, as the DLS and NMR experiments were conducted on different batches of precursor polymer (which showed virtually identical molecular weight and comonomer content). Aminomethyl styrene is extremely sensitive to deactivation by $\mathrm{CO}_{2}$. Even though the samples were handled with care under inert gas atmosphere, differences between the samples cannot be fully excluded, and the desirable synchronous measurement of parts of the same sample solution in the different instruments was not possible in the framework of this study. By comparing DLS studies of different polymer batches, statistical errors in the gel point time were estimated and are indicated by the error bars in Figure 6. The smaller error bars associated with the NMR data do not reflect the statistical batchto-batch variation, but indicate the error associated with the fitting of the noisy data in Figure 5b.

However, true deviations between NMR and DLS as observed here could be explained as follows: The appearance of a speckle in scattering from gels is an interference phenomenon whose associated length scale is on the order of $50 \mathrm{~nm}$. In addition, the integration time for a single point is $20 \mathrm{~s}$. Thus, DLS probes structures on somewhat larger length scales and longer time scales than NMR, the latter being sensitive to fast single-network chain fluctuations that retain some anisotropy in the ms range. The difference is thus not surprising, and we emphasize the strong complementarity of the results that ultimately lead to a better understanding of gel formation. In another study on polysaccharides, DLS was also found to detect gelation later than rheology, and possible reasons are discussed at length in ref 44.

The apparent gelation time constant, and thus the rate of network formation, is found to be virtually independent of concentration, which is in fact expected for a (probably diffusion-controlled) first-order process. We found that both the gelation time constant as well as the gel point time decreased by about a factor of 2 when performing the experiments at $T=$ $35^{\circ} \mathrm{C}$. In agreement with the detailed report on the DLS studies of this system, ${ }^{8,42}$ no gelation is detectable at concentrations below $0.02 \mathrm{~g} / \mathrm{mL}$. This is explained by the fact that the overlap concentration of the prepolymer is around $0.01 \mathrm{~g} / \mathrm{mL}$ (thus the investigated solutions are strictly semidilute), around and below which only microgels are formed. These reorient too quickly to produce observable chain order (residual couplings) on the NMR time scale.

Finally, we focus on the in-depth NMR analysis of the final gels to again stress the rich insight that is gained by combination 


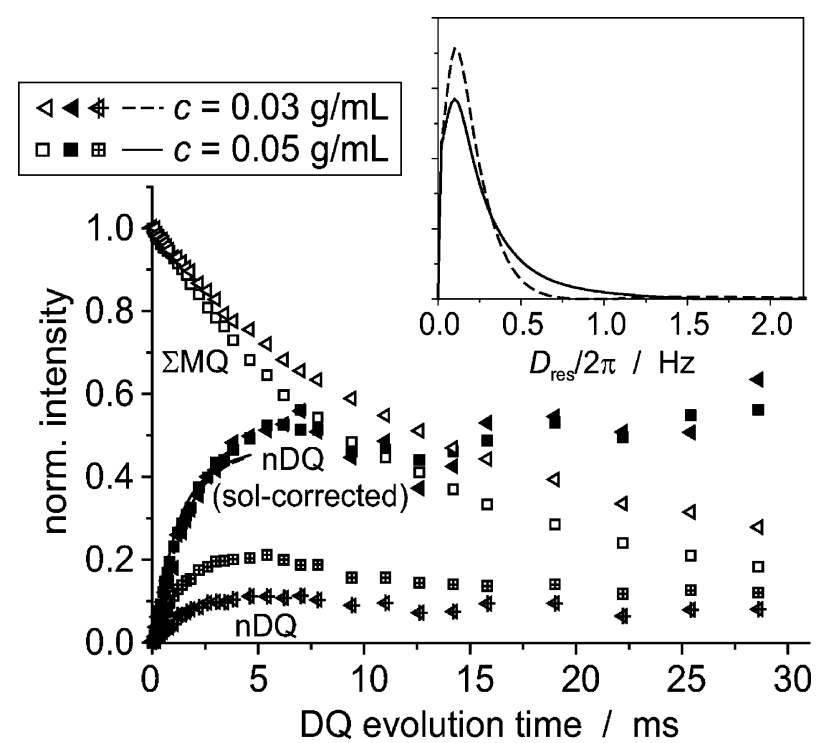

Figure 7. Final PS gel characterization by DQ build-up analysis. Crossed symbols represent the direct experimental $I_{\mathrm{nDQ}}$, and solid symbols result after subtraction of two mobile components $\mathrm{B}$ and $\mathrm{C}$ from $I_{\Sigma \mathrm{MQ}}$ before normalization. The so-obtained $I_{\mathrm{nDQ}}$ were subjected to numerical analysis of the underlying broad distribution of residual dipolar couplings (see inset).

Table 1. Results of the Final MQ NMR Gel Characterization of the Solution-Cross-Linked P(S-co-AMS $)^{a}$

\begin{tabular}{|c|c|c|c|c|c|c|c|c|}
\hline $\begin{array}{c}c \\
\mathrm{~g} / \mathrm{mL}\end{array}$ & $f_{\mathrm{C}}$ & $f_{\mathrm{tolH}}$ & $\begin{array}{r}T_{2 \mathrm{C}} \\
\mathrm{ms}\end{array}$ & $f_{\mathrm{B}}$ & $\begin{array}{r}T_{2 \mathrm{~B}} \\
\mathrm{~ms}\end{array}$ & $\begin{array}{l}f_{\mathrm{A}} \\
\mathrm{Hz}\end{array}$ & $\begin{array}{c}D_{\text {reg }} / 2 \pi \\
\mathrm{Hz}\end{array}$ & $\sigma_{\text {reg }} / 2 \pi$ \\
\hline 0.02 & 0.31 & 0.10 & 82 & 0.56 & 13.6 & 0.13 & 278 & 248 \\
\hline 0.03 & 0.29 & 0.07 & 71 & 0.45 & 13.0 & 0.26 & 194 & 185 \\
\hline 0.04 & 0.17 & 0.05 & 85 & 0.45 & 17.5 & 0.38 & 199 & 187 \\
\hline 0.05 & 0.07 & 0.04 & 129 & 0.43 & 18.1 & 0.50 & 229 & 202 \\
\hline
\end{tabular}

${ }^{a}$ Corresponding data is shown in Figure 7. The inevitable residual protonated toluene fraction is detected as part of the mobile component $\mathrm{C}$ and is estimated from pure solvent FID intensities, scaled to the varying overall polymer intensity.

with DLS. Figure 7 and Table 1 summarize the NMR results. Notably, apart from the network component A there are now two exponential contributions to the $\Sigma \mathrm{MQ}$ decays of the gels that can be reliably separated. Component $\mathrm{C}$ is very mobile, with an apparent $T_{2 \mathrm{C}}$ on the order of $100 \mathrm{~ms}$. Its fraction decreases from $30 \%$ down to $7 \%$ with increasing polymer concentration; note that about $1 / 3$ of this component is composed of residual protons in the deuterated toluene. The rest of this component probably corresponds to the extractable sol, which should amount to $10 \%$.

The fraction of the second, less mobile component $\left(T_{2 \mathrm{~B}} \approx\right.$ $13-18 \mathrm{~ms}$ ) is on the order of $50 \%$ and almost constant. It does not contribute to the generation of DQ intensity, and it is therefore either attributed to network defects like dangling chains and loops or to starlike structures or microgels that all have close-to-isotropic larger-scale chain dynamics (overall rotational diffusion or arm retraction). These structures are part of or trapped in the network structure but do not contribute to the elasticity. Finally, the amount of polymer that is ultimately converted into elastically active network rises from about 13 to $50 \%$. All these findings are in agreement with the rather low cross-linking efficiency in these systems $(3-8 \%)$ that was derived from the shear moduli. ${ }^{42}$

The DQ build-up data shown in Figure 7, normalized after the subtraction of the mobile fractions, reach their expected plateau at $I_{\mathrm{nDQ}} \approx 0.5$, proving that the amount of subtracted $\mathrm{B}$ and $\mathrm{C}$ components is roughly correct. The underlying distribu- tion of residual couplings shown in the inset is broad (indicated by the nonparabolic initial and convex overall shape of $I_{\mathrm{nDQ}}$ ) and could only be estimated using a numerical inversion procedure. ${ }^{32}$ The structure thus exhibits considerable heterogeneities, as we also have found in a study of swollen, bulk endlinked PDMS gels. ${ }^{34}$ How far the heterogeneities formed upon cross-linking in solution and upon swelling of bulk cross-linked systems are related remains the subject of further work. Note that this type of polydispersity, detected at the molecular level, is not easily accessible by any other method.

The most important result of the build-up analysis is that, within the given error limits posed by the weak signal in (semi)dilute solution, all gels have a similar apparent chain length between cross-links or trapped entanglements, irrespective of concentration. This is of course expected for chains with branch points that are predefined by the randomly placed amino functionalities, and this is to be compared with the complementary result of the DLS intensity time correlation function analysis that yields power-law exponents for the slow mode related to cooperative diffusion in the gel. ${ }^{8}$ This exponent, also accessible by rheological experiments, is commonly attributed to the fractal dimension of the network, and this structural parameter was also found to be neither a function of concentration nor of gel point time (the latter aspect, gel structure analysis during the gelation run, was not done by NMR in the present context but is of course highly worthwhile). Therefore, NMR nicely complements the DLS analysis with information on the local network structure on the length scale of individual network chains.

Mechanical measurements gave shear moduli that increased from 300 to about $1500 \mathrm{~Pa}$ over the investigated series. ${ }^{42}$ This is to be compared with the case of unswollen elastomers that always show a linear correspondence between the modulus and the NMR observable. ${ }^{36,45-49}$ Here, one of course has to take into account that the network fraction $\mathrm{A}$ as well as the overall polymer volume fraction both increase over the sample series. The apparent contradiction can thus be explained because the modulus is inversely proportional to the volume fraction of polymer. ${ }^{50}$ However, our first NMR investigations of partially swollen systems showed surprising results with regard to swelling heterogeneities and the overall weak swelling effect on chain stretching and entropy loss, ${ }^{34}$ and an in-depth correlation of mechanical and NMR properties of networks in the swollen state is yet to be established.

\section{Conclusions}

In summary, we have shown that the mere observation of DQ intensity, arising from chain motions that are nonisotropic on the ms time scale, is a sensitive marker of the onset of gelation in bulk as well as dissolved systems with weakly entangled precursor polymers. In end- and randomly cross-linked bulk PDMS of moderate initial molecular weight and varying contents of cross-linker, the so-determined gel point, was found to be essentially identical to the results of much more timeconsuming rheological experiments. In randomly cross-linked solutions of functionalized PS with stoichiometric amounts of cross-linker, the NMR-detected gel point time also followed the same trend as the time at which speckles appeared in DLS experiments. Weak deviations toward shorter NMR-detected gel point times are in line with the notion that NMR is sensitive to gelation on a shorter length and time scale.

Further work will have to show how this approach has to be adapted to the case of long, entangled prepolymers that exhibit significant entanglement-induced residual dipolar couplings even when no cross-links are present. ${ }^{26,30,38}$ One might envision the 
observation of a discontinuity in $I_{\mathrm{nDQ}}$ at the gel point, yet, as entanglement-induced residual couplings are a strong function of temperature, ${ }^{38}$ an extended protocol might necessitate a study at different temperatures. Cohen-Addad has discussed other possible approaches along these lines based on proton transverse relaxation properties; ${ }^{26}$ the application of MQ spectroscopy to this type of system is underway.

The NMR approach offers the advantage that it offers at least semiquantitative insight into the gelation process on the molecular scale, i.e., the contents of mobile fractions, the network structure, and the chain mobility can be monitored during the course and at the end of gelation. Our results complement and support results from rheological and light scattering experiments, where in particular for cross-linking in solution, the observation of a concentration-independent chain length between cross-links nicely complements the knowledge on the constant fractal dimension of the gel as detected by DLS.

The NMR methodology presented herein should be applicable to all polymeric systems, bulk or dilute, that still retain a high chain mobility and flexibility in the gel state. In other works, the systems should be far above $T_{\mathrm{g}}$. This is a necessary condition to observe the gelation-induced (single-point) DQ intensity at sufficiently long DQ times without having to worry about the overall intensity relaxation. Thermoset gelation, where $T_{\mathrm{g}}$ of the final product rises above the gelation temperature, as well as the formation of physical gels, where cross-links are often formed by solid-like aggregates or even (nano)crystalline structures, are problematic cases, as the spectral intensity associated with the network fraction relaxes very quickly. Significant parts of the sample might even escape detection due signal loss during the MQ pulse sequence and the receiver dead time. Transverse relaxation methods have been applied to such systems, ${ }^{16,51,52}$ but the phenomenology is diverse and must be carefully established for each case.

Limitations also apply to mixed multicomponent systems such as block copolymers, blends, or solutions using nondeuterated solvents. At low field, one must then make sure that the observed intensity stems only from the component in which the crosslinking takes place, either by careful component analysis or by implementing magnetization filters (e.g., simple transverse dephasing of rigid-phase magnetization). Another option is of course to take advantage of the (limited) chemical-shift resolution provided by static experiments performed at high field ${ }^{36}$ or to use DQ methods that work under conditions of fast magicangle spinning ${ }^{30,53}$ in order to detect DQ intensities selectively for a certain chemical species.

Acknowledgment. Funding of this work was provided by the Deutsche Forschungsgemeinschaft (SFBs 418 and 428) and the Fonds der Chemischen Industrie.

\section{References and Notes}

(1) Adam, M.; Lairez, D. Sol-Gel Transition. In The Physical Properties of Polymeric Gels; Cohen-Addad, J. P., Ed.; John Wiley \& Sons: New York, 1996, pp 87-142.

(2) Chambon, F.; Winter, H. H. Polym. Bull. 1985, 13, 499-503.

(3) Winter, H. H.; Chambon, F. J. Rheol. 1986, 30, 367-382.

(4) Chambon, F.; Winter, H. H. J. Rheol. 1987, 31, 683-697.

(5) Martin, G. E.; Zektzer, A. S. Two Dimensional NMR Methods for Establishing Molecular Connectivity; VCH: Weinheim, 1988.

(6) Martin, J. E.; Wilcoxon, J. P.; Odinek, J. Phys. Rev. A 1991, 43, 858872.

(7) Shibayama, M.; Norisuye, T. Bull. Chem. Soc. Jpn. 2002, 75, 641659.

(8) Liu, R. G.; Gao, X.; Oppermann, W. Polymer 2006, 47, 8488-8494.

(9) Stöver, H. D. H.; Fréchet, J. M. J. Macromolecules 1991, 24, 883888 .
(10) Hansen, E. W.; Holm, K. H.; Jahr, D. M.; Olafsen, K.; Stori, A. Polymer 1997, 38, 4863-4871.

(11) Richter, S.; Brand, T.; Berger, S. Macromol. Rapid Commun. 2005, 26, 548-553.

(12) Brand, T.; Richter, S.; Berger, S. J. Phys. Chem. B 2006, 110, 1585315857.

(13) Folland, R.; Charlesby, A. Int. J. Radiat. Phys. Chem. 1976, 8, 555562.

(14) Charlesby, A.; Folland, R.; Steven, J. H. Proc. R. Soc. London, Ser. A 1977, 355, 189-207.

(15) Cohen-Addad, J. P. Progr. NMR Spectrosc. 1993, 25, 1-316.

(16) Chiu, H. T.; Wang, J. H. Polymer 1999, 40, 6859-6864.

(17) Hong, P. D.; Chou, C. M.; Chuang, W. T. J. Appl. Polym. Sci. 2001, 79, 1113-1120.

(18) Cohen-Addad, J. P. J. Chem. Phys. 1973, 60, 2440-2453.

(19) Cohen-Addad, J. P. Macromolecules 1989, 22, 147-151.

(20) Cohen-Addad, J. P.; Vogin, R. Phys. Rev. Lett. 1974, 33, 940-943.

(21) Collignon, J.; Sillescu, H.; Spiess, H. W. Colloid Polym. Sci. 1981, 259, 220-226.

(22) Chapter G. Fry; Lind, A. C. Macromolecules 1988, 21, 1292-1297.

(23) Simon, G.; Baumann, K. and Gronski, W. Macromolecules 1992, 25, 3624-3628.

(24) Callaghan, P. T.; Samulski, E. T. Macromolecules 1997, 30, 113-122.

(25) Fischer, E.; Grinberg, F.; Kimmich, R.; Hafner, S. J. Chem. Phys. 1998, 109, 846-854.

(26) Cohen-Addad, J. P.; Soyez, E.; Viallat, A.; Queslel, J. P. Macromolecules 1992, 25, 1259-1266.

(27) Cohen-Addad, J. P.; Montes, H. Macromolecules 1997, 30, 36783684.

(28) Litvinov, V. M. Characterization of Chemical and Physical Networks in Rubbery Materials Using Proton NMR Magnetization Relaxation. In Spectroscopy of Rubbers and Rubbery Materials; Litvinov, V. M. De, P. P., Eds.; Rapra Technology Ltd.: Shawbury, U.K. 2002; Chapter 10, pp 353-400.

(29) Baum, J.; Munowitz, M.; Garroway, A. N.; Pines, A. J. Chem. Phys. 1985, 83, 2015-2025

(30) Graf, R.; Heuer, A.; Spiess, H. W. Phys. Rev. Lett. 1998, 80, 57385741.

(31) Schneider, M.; Gasper, L.; Demco, D. E.; Blümich, B. J. Chem. Phys. 1999, 111, 402-415.

(32) Saalwächter, K.; Ziegler, P.; Spyckerelle, O.; Haidar, B.; Vidal, A.; Sommer, J. U. J. Chem. Phys. 2003, 119, 3468-3482.

(33) Wiesmath, A.; Filip, C.; Demco, D. E.; Blümich, B. J. Magn. Reson. 2002, 154, 60-72.

(34) Saalwächter, K.; Kleinschmidt, F.; Sommer, J. U. Macromolecules 2004, 37, 8556-8568

(35) Saalwächter, K.; Klüppel, M.; Luo, H.; Schneider, H. Appl. Magn. Reson. 2004, 27, 4071-417.

(36) Saalwächter, K.; Herrero, B.; López-Manchado, M. A. Macromolecules 2005, 38, 9650-9660

(37) Saalwächter, K. J. Chem. Phys. 2004, 120, 454-664.

(38) Saalwächter, K.; Heuer, A. Macromolecules 2006, 39, 3291-3303.

(39) Saalwächter, K. Macromolecules 2005, 38, 1508-1512.

(40) Saalwächter, K.; Herrero, B.; López-Manchado, M. A. Macromolecules 2005, 38, 4040-4042.

(41) Saalwächter, K. J. Am. Chem. Soc. 2003, 125, 14684-14685.

(42) Liu, R. G.; Oppermann, W. Macromolecules 2006, 39, 4159-4167.

(43) Clarson, S. J.; Wang, Z.; Mark, J. E. Eur. Polym. J. 1990, 26, 621622.

(44) Richter, S.; Matzker, R.; Schröter, K. Macromol. Rapid Commun. 2005 , $26,1626-1632$.

(45) Simon, G.; Götschmann, B.; Matzen, D.; Schneider, H. Polym. Bull. 1989, 21, 475-482.

(46) Demco, D. E.; Hafner, S.; Fülber, C.; Graf, R.; Spiess, H. W. J. Chem. Phys. 1996, 105, 11285-11296.

(47) Sotta, P.; Fülber, C.; Demco, D. E.; Blümich, B.; Spiess, H. W Macromolecules 1996, 29, 6222-6230.

(48) Cohen-Addad, J. P.; Thanh, B. P.; Montes, H. Macromolecules 1997, 30, 4374-4380.

(49) Saalwächter, K. Progr. NMR Spectrosc. 2007, in press.

(50) Flory, P. J. Principles of Polymer Chemistry; Cornell University Press: Ithaca, New York, 1953.

(51) Hietalahti, K.; Root, A.; Skrifvars, M.; Sundholm, F. J. Appl. Polym. Sci. 1997, 65, 77-83.

(52) Jahromi, S.; Litvinov, V.; Geladé, E. J. Polym. Sci., Part B: Polym. Phys. 1999, 37, 3307-3318.

(53) Graf, R.; Demco, D. E.; Hafner, S.; Spiess, H. W. Solid State Nucl. Magn. Reson. 1998, 12, 139-152.

MA062776B 